\title{
Effects of low-dose tacrolimus therapy in combination with methotrexate in patients with methotrexate-refractory rheumatoid arthritis
}

This article was published in the following Dove Press journal:

Open Access Rheumatology: Research and Reviews

3 July 2010

Number of times this article has been viewed

\author{
Takeo Isozaki \\ Michihito Sato \\ Ryo Takahashi \\ Kuninobu Wakabayashi \\ Nobuyuki Yajima \\ Yusuke Miwa \\ Masao Negishi \\ Hirotsugu Ide \\ Tsuyoshi Kasama
}

Division of Rheumatology, Department of Medicine, Showa University School of Medicine, Tokyo, Japan
Correspondence:Takeo Isozaki Department of Medicine, Showa University School of Medicine, I-5-8 Hatanodai, Shinagawa-ku, Tokyo I42-8666, Japan

Tel +8I 337848942

Fax +8I 337848946

Email t.isozaki@med.showa-u.ac.jp

\begin{abstract}
The aim of the present clinical trial was to determine the efficacy and safety of low-dose administration of tacrolimus in combination with methotrexate (MTX) in rheumatoid arthritis (RA) patients with an insufficient clinical response to MTX alone. Eleven patients with active RA, despite treatment with MTX, were enrolled and given tacrolimus in combination with MTX for 24 weeks. The primary endpoint was the assessment of clinical improvement using the European League against Rheumatism criteria. Administration of tacrolimus to RA patients with an insufficient response to MTX produced significant improvement in the Disease Activity Score 28 after 8-24 weeks. In addition, after 24 weeks, $50 \%$ and $25 \%$ of patients had achieved moderate and good responses, respectively, and there were significant reductions in the Modified Health Assessment Questionnaire, the rheumatoid factor and serum matrix metalloproteinase-3 levels. The present preliminary study suggests that low-dose tacrolimus in combination with MTX is well tolerated and provides both clinical and economic benefits.
\end{abstract}

Keywords: rheumatoid arthritis, methotrexate, tacrolimus, DAS28, mHAQ

\section{Introduction}

Rheumatoid arthritis (RA) is a chronic, progressive disease that requires early diagnosis and aggressive treatment to minimize morbidity. ${ }^{1,2}$ Disease-modifying antirheumatic drugs (DMARDs) are used to reduce inflammation and slow the progression of articular damage. Methotrexate (MTX) is the most widely used DMARD because of its favorable efficacy, record of safety, and ability to produce a prolonged response. ${ }^{3,4}$ Many patients, however, have an insufficient clinical response to MTX. In those patients, biologics such as infliximab or etanercept are used along with MTX. Although biologics are beneficial for patients with partial responses to MTX, their high cost makes them unobtainable for many RA patients. In these patients, combination therapies of MTX and other DMARDs have been considered.

Tacrolimus, a calcineurin inhibitor, was developed as an immunosuppressive agent and has been used in patients following organ transplantation, as well as in those with rheumatoid arthritis (RA) in Japan. Tacrolimus, a novel immunosuppressant, has been isolated from the fermentation broth of Streptomyces tsukubaenis $\mathrm{Nr} 9993$ as a colorless prism, and the molecular formula was determined to be C44H69NO12.H2O. The compound suppresses immune responses in vitro and in vivo in mice ${ }^{5}$ and inhibits $T$ cell activation, thereby suppressing the production of tumor necrosis factor- $\alpha$ and other inflammatory cytokines involved in the development of RA. ${ }^{6}$ FK506 (tacrolimus) inhibits both interleukin-2 and gamma-interferon secretion of PBMCs stimulated 
with PHA in a dose-dependent manner. ${ }^{7}$ Yamagami $\mathrm{T}$ et al showed a novel mechamism for the action of physiological concentrations of FK506 in RA that regulates the production of glistatin/thymidine phosphorylase in fibroblast-like synoviocytes. ${ }^{8}$ There are many reports about the efficacy and safety to RA patients. ${ }^{9-11}$ Moreover, there is also a report to systemic lupus erytematosus, ${ }^{12}$ Churg-Strauss symdrome, ${ }^{13}$ and the RA associated with MDS. ${ }^{14}$

To reduce the economic cost and the risk of immoderate immune suppression from combination therapy, it would be beneficial to examine the efficacy of therapy with low-dose administration of tacrolimus in combination with MTX in patients with RA. The aim of the present clinical trial was to determine the efficacy and safety of low-dose tacrolimus in combination with MTX to control the signs and symptoms of RA in patients with an insufficient clinical response to MTX alone.

\section{Methods}

\section{Study design and population}

Eleven patients who fulfilled the American College of Rheumatology (ACR) 1987 revised criteria for RA ${ }^{15}$ between June 2009 and November 2009 participated in this prospective, open, nonplacebo-controlled study. RA disease activity was evaluated using the Disease Activity Score (DAS) 28. ${ }^{16}$ Patients who had moderate or severe disease activity (DAS28 > 3.2) at baseline despite treatment with MTX for more than 3 months were enrolled and given tacrolimus. Tacrolimus is typically given at a dosage of $3 \mathrm{mg} /$ day to patients with RA. In the present study, we used a starting dosage of $1 \mathrm{mg} /$ day. Our purpose was to examine the combined use in low dose $(1 \mathrm{mg})$, and when an effect was insufficient, patients changed to an other treatment. All other DMARDs were discontinued except MTX, but stable doses of nonsteroidal anti-inflammatory drugs and oral corticosteroids ( $<10 \mathrm{mg}$ /day prednisone or its equivalent) were allowed. When DAS28 $<2.6$ was achieved, prednisolone started dose down by $1 \mathrm{mg}$.

The primary endpoint was the assessment of clinical improvement in the DAS28 using the European League against Rheumatism (EULAR) response criteria. ${ }^{17}$ Secondary endpoints were the assessment of changes in the Modified Health Assessment Questionnaire (mHAQ), ${ }^{18}$ serum levels of rheumatoid factor (RF) and matrix metalloproteinase-3 (MMP-3), and the incidence of remission and adverse events. Serum levels of C-reactive protein (CRP), RF, and MMP-3 were determined using latex photometric immunoassays, while the erythrocyte sedimentation rate (ESR) was determined using the Westergren method. At baseline, demographic data and present medications were recorded; a complete history was taken; and a physical examination and routine laboratory examinations, including whole blood counts, tests of renal and hepatic function, and urinalysis, were carried out at 3- to 6-week intervals to screen for adverse clinical side effects. The aforementioned clinical parameters were assessed at baseline and after 12 and 24 weeks of tacrolimus-MTX combination therapy. The present clinical trial was performed in accordance with protocols approved by the Human Subjects Research Committee at our institution, and informed consent was obtained from all patients.

\section{Statistical analysis}

The data are expressed as the mean \pm standard error (SE). For a comparison of the groups, chi-square analysis was applied for discrete variables, and the Kruskal-Wallis analysis of variance (ANOVA) was used for continuous variables. Changes in variables after intervention were evaluated using the Wilcoxon test for paired observations. $P$ values less than 0.05 were considered significant.

\section{Results}

The patient characteristics are summarized in Table 1. At the start of this trial, the mean age of the patients was 65.5 (range: 49-82) years, and disease duration was 8.8 (0.5-14) years. Baseline DAS28 for the 11 patients was 4.51 (2.95-6.17); MTX dosage and duration were 8.73 (8-12) $\mathrm{mg} /$ week and $3.18(0.5-10)$ years, respectively; and mHAQ was $0.58(0.125-1)$. The dosage of MTX administered to our patients was somewhat lower than that reported in other countries; this likely reflects the lower effective and recommended dosages of MTX (6-8 mg per week) in Japanese RA patients, which is in accordance with the results of a double-blind, placebo-controlled (phase II-III) trial among Japanese RA patients. Adverse events were observed in one RA patient with diabetes mellitus which was discontinued after 8 weeks because of a mild increase in blood glucose levels. The patient soon recovered without any additional

Table I Clinical characteristics of the patients enrolled in patient trial

\begin{tabular}{ll}
\hline Mean age (years) & $65.5(49-82)$ \\
Sex (male/female) & $\mathrm{II}(\mathrm{I} / \mathrm{I} 0)$ \\
Steinbrocker stage & stage I-2, II-3, III-6 \\
Steinbrocker class & class $2-9,3-2$ \\
Disease duration (years) & $8.8(0.5-\mathrm{I} 4)$ \\
Prednisolone (mg/day) & $1.64(0-5)$ \\
MTX dosage (mg/week) & $8.73(8-12)$ \\
Mean DAS28 (ESR4) & $4.45(2.95-6.17)$ \\
Mean mHAQ & $0.58(0.125-\mathrm{I})$ \\
\hline
\end{tabular}

Notes: Results shown are mean (range). 
medications. Furthermore, treatment was discontinued in one patient at 4 weeks, in one patient at 8 weeks, and in one patient at 20 weeks due to increases in RA disease activity or a lack of response to the combination therapy. Ten patients spent 8 weeks; nine patients spent 20 weeks; and eight patients spent 24 weeks receiving the combination therapy and completed the assessments and clinical examinations. The RA patients with an insufficient response to MTX who received the tacrolimus-MTX combination showed highly significant improvement in the DAS28 after 4 weeks $(\mathrm{n}=10$, baseline: $4.51 \pm 0.31$ to $4.03 \pm 0.28, P<0.005)$, 12 weeks (n $=9$, baseline: $4.59 \pm 0.34$ to $3.38 \pm 0.29, P<0.01$ ) and 24 weeks ( $\mathrm{n}=8$, baseline: $4.45 \pm 0.35$ to $3.12 \pm 0.34$, $P<0.01$ ) (Figure 1). In addition, after 12 weeks, $44.4 \%$ of patients achieved a moderate response based on the EULAR criteria, and after 24 weeks, the number of those patients were maintained (Table 2). There were no significant differences between the poor response group and the moderate-good response group with respect to mean disease duration, MTX dosage or basal DAS28 and CRP levels (data not shown). Notably, clinical remission (DAS28 < 2.6) was observed in one RA patient after 24 weeks of combination therapy. In addition to the DAS28, significant reductions in serum CRP levels were observed after 12 weeks $(n=9$, baseline: $1.92 \pm 0.70$ to $0.86 \pm 0.34, P<0.05)$ and 24 weeks $(\mathrm{n}=8$, baseline: $2.09 \pm 0.77$ to $0.51 \pm 0.18, P<0.01$ ) (Figure 2 ). Moreover, there were significant improvements in mHAQ

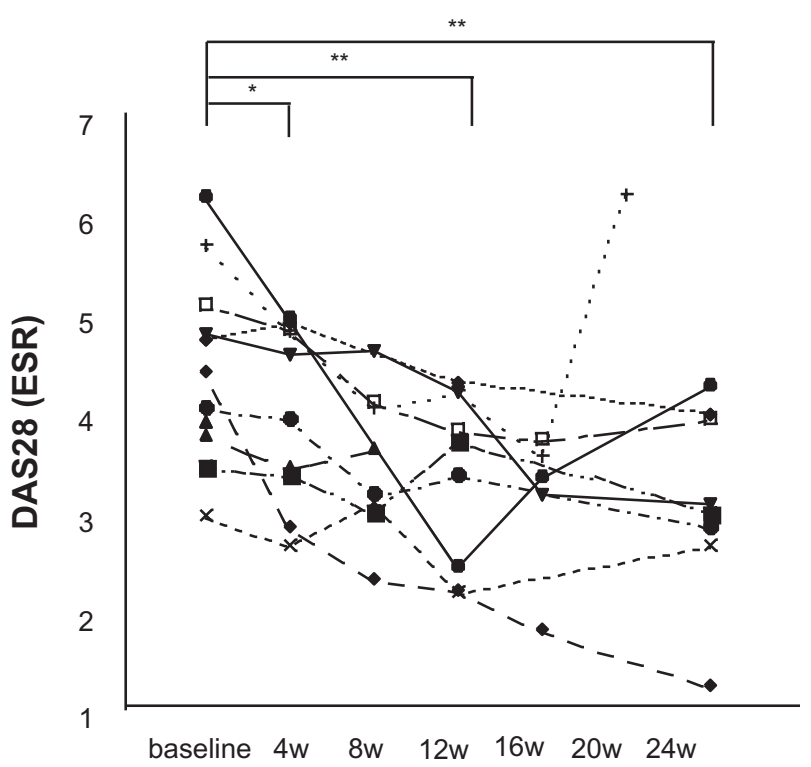

Figure I Effect of tacrolimus-MTX combination therapy on the DAS28 in patients with RA. The DAS28 in RA patients was assessed from baseline to 24 weeks after the addition of tacrolimus to MTX therapy. Significant improvements in the DAS28 were seen at 4 weeks $(* P<0.005)$ and after $12-24$ weeks $(* * P<0.01)$ of tacrolimus-MTX combination therapy compared with baseline.
Table 2 Percentage of patients who achieved moderate and good response in the EULAR response criteria

\begin{tabular}{lll}
\hline Response & I 2 weeks $(\mathbf{n}=\mathbf{9})$ & 24 weeks $(\mathbf{n}=\mathbf{8})$ \\
\hline Good response & $2(22.2 \%)$ & $2(25 \%)$ \\
Moderate response & $4(44.4 \%)$ & $4(50 \%)$ \\
No response & $3(33.3 \%)$ & $2(25 \%)$ \\
\hline
\end{tabular}

Notes: Clinical improvement in RA was assessed using the EULAR criteria based on the change of DAS28.

responses after both 12 weeks $(n=9$, baseline: $0.54 \pm 0.10$ to $0.24 \pm 0.06, P<0.01)$ and 24 weeks $(\mathrm{n}=8$, baseline: $0.58 \pm 0.11$ to $0.20 \pm 0.06, P<0.01$ ), with $75 \%$ of the patients experiencing 50\% improvement in their mHAQ responses after 12 and 24 weeks (Figure 3 ).

\section{Changes in the levels of serum parameters}

We next assessed changes in serum RF and MMP-3 levels. There was a significant reduction in serum RF levels after 24 weeks ( $\mathrm{n}=6$, baseline: $82.6 \pm 27.1$ to $50.2 \pm 15.1, P<0.05$ ) (Figure 4A). Similarly, there was a significant reduction in serum MMP-3 levels after 24 weeks $(n=5$, baseline: $407.7 \pm 137.0$ to $80.0 \pm 21.9, P<0.05$ ) (Figure 4B).

\section{Dosage of medicine}

We were able to decrease the dosage of oral corticosteroids in two patients. The dosage for one patient was reduced from $4 \mathrm{mg}$ to $2 \mathrm{mg}$ after 24 weeks, and the dosage for another patient was

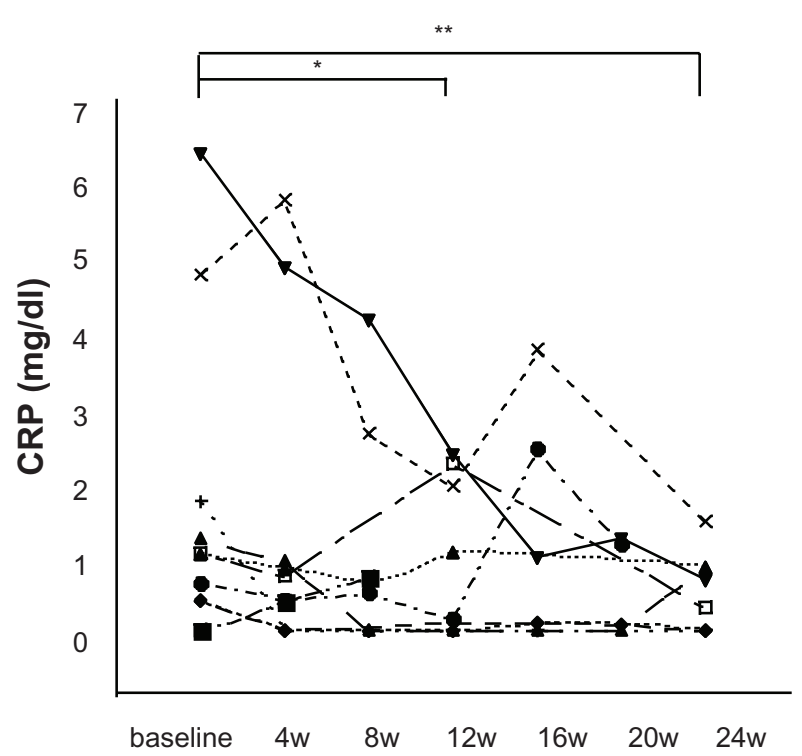

Figure 2 Effect of tacrolimus-MTX combination therapy on serum CRP levels in patients with RA. Serum CRP levels were assessed from baseline to 24 weeks after the addition of tacrolimus to MTX therapy. Significant reductions in serum CRP levels were seen after 12 weeks $(* P<0.05)$ and 24 weeks $(* * P<0.0$ I) of tacrolimus-MTX combination therapy compared with baseline. 


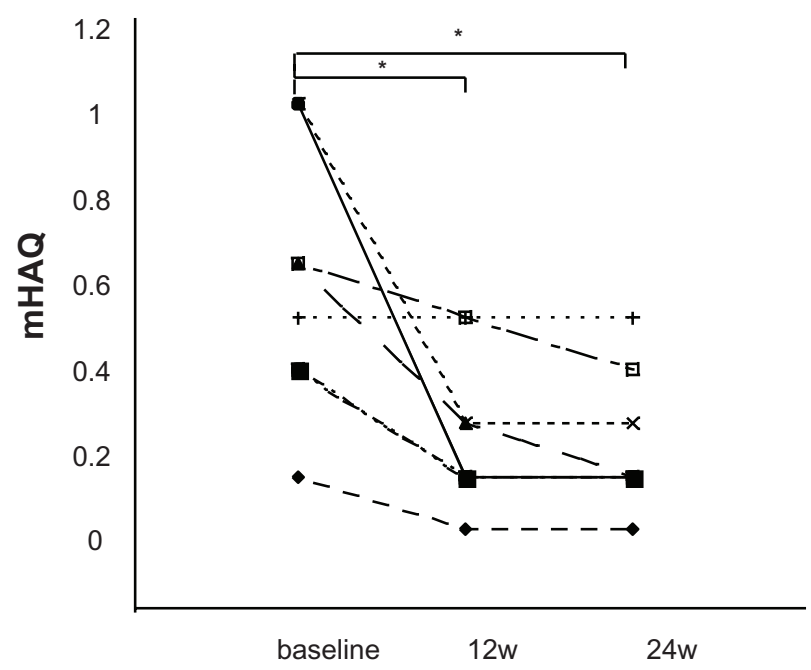

Figure 3 Effect of tacrolimus-MTX combination therapy on $\mathrm{mHAQ}$ responses in patients with RA. Significant improvements in $\mathrm{mHAQ}$ responses were seen after both $12(* P<0.01)$ and $24(* P<0.01)$ weeks of tacrolimus-MTX therapy.

reduced from $1 \mathrm{mg}$ to nothing after 24 weeks. The dosages of any other medicines (MTX and nonsteroidal anti-inflammatory drugs) remained the same before and after treatment.

\section{Discussion}

The data presented in this study show that the administration of a tacrolimus-MTX combination to RA patients with an insufficient response to MTX led to a highly significant improvement of the DAS28 and the EULAR response criteria after 8-24 weeks (Figure 1). In addition, there was a significant improvement in $\mathrm{mHAQ}$ responses, suggesting beneficial effects on quality of life. ${ }^{19}$ We also found significant serological effects following tacrolimus administration, including a significant reduction in MMP-3 levels after 24 weeks. Given earlier findings that elevated serum MMP-3 levels may contribute to the progression of joint destruction, ${ }^{20,21}$ we suggest that tacrolimus-MTX combination therapy may exert a beneficial effect on the clinical course of patients with active RA.

After failure with MTX, combination therapy with other DMARDs or biologics is considered. ${ }^{22}$ Although sulfasalazine (SSP) is widely used in combination with MTX because they are both inexpensive and readily available to everyone, there are conflicting results with regard to their efficacy. Whereas Capell et al showed that SSP-MTX combination therapy provides significant beneficial clinical effects compared with MTX monotherapy, ${ }^{23}$ others have reported that SSP provides no beneficial additive or synergistic effects in combination with MTX. ${ }^{24}$ Additionally, although clinical trials of combination therapies with MTX plus other immunosuppressants or strong DMARDs, including cyclosporine A (CsA) and leflunomide, have shown beneficial

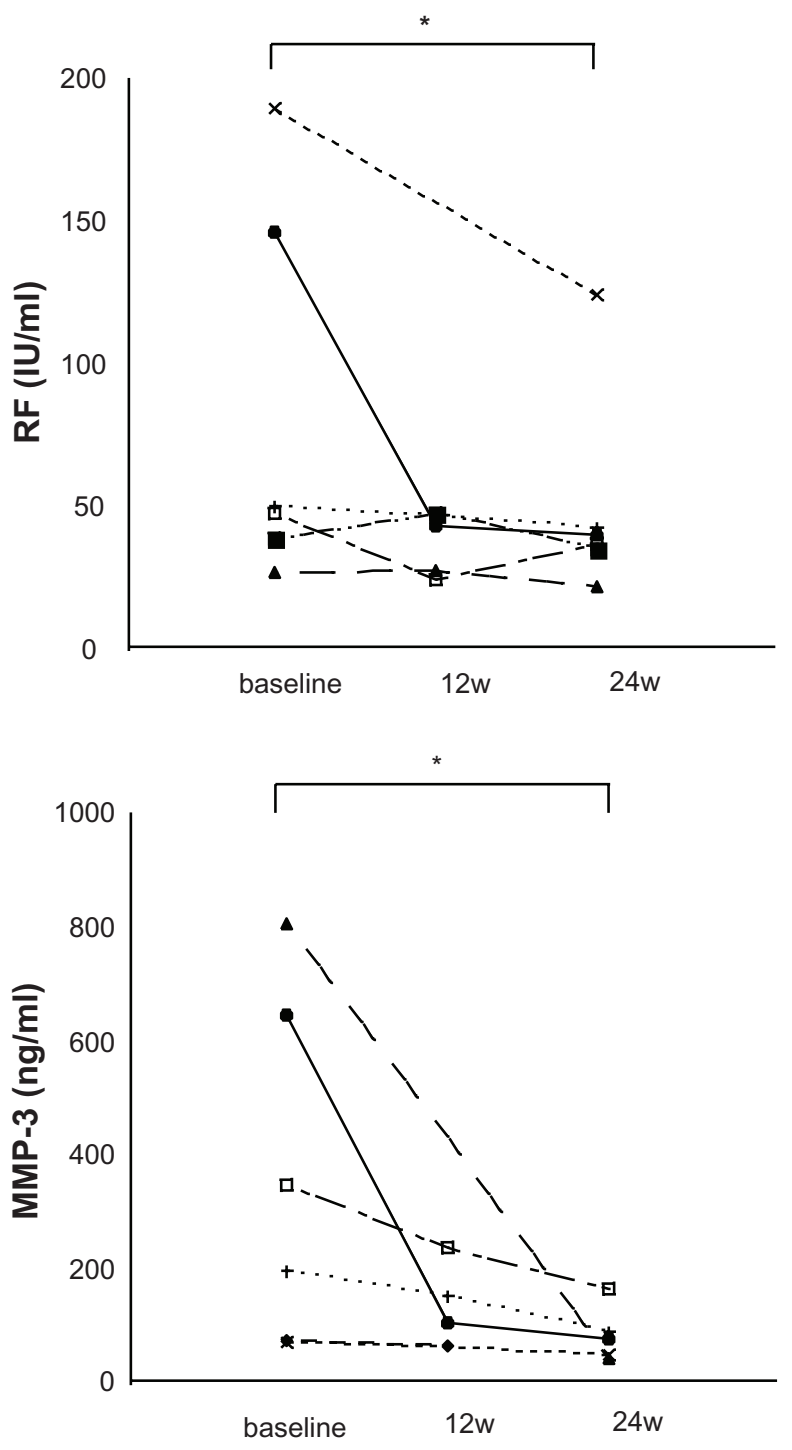

Figure 4 Effect of tacrolimus-MTX combination therapy on serum RF levels and serum MMP-3 levels in patients with RA. A) Serum RF levels and serum MMP-3 levels were assessed at baseline and 12 and 24 weeks after the addition of tacrolimus to MTX therapy. Significant reductions ( $* P<0.05$ ) in serum RF levels were seen after 24 weeks of tacrolimus-MTX combination therapy compared with baseline. B) Significant reductions $(* P<0.05)$ in serum MMP-3 levels were seen after 24 weeks of tacrolimus-MTX combination therapy compared with baseline.

effects in RA patients with high disease activity, there is an increased risk of adverse effects with these generally acting drugs. ${ }^{25,26}$ Kasama et al showed that low-dose mizoribine in combination with MTX is well tolerated and provides clinical benefits. ${ }^{27}$ In contrast, another study administered tacrolimus at $3 \mathrm{mg} /$ day to $80 \mathrm{RA}$ patients with high disease activity and found an inadequate effect of MTX. Seven patients $(12.5 \%)$ withdrew from the study because of adverse events, possibly related to treatment with tacrolimus. ${ }^{28}$ One study administered tacrolimus at $1-3 \mathrm{mg}$ per day to RA patients with an inadequate response to MTX and found significant improvement in the DAS28 (ESR), DAS28 (CRP), tender 
Table 3 Reports of clinical efficacy of tacrolimus therapy in RA patients with an insufficient response to MTX

\begin{tabular}{|c|c|c|c|c|}
\hline References & No of patients & Dosage of tacrolimus & Assessments of efficacy & Efficacy of MTX-tacrolimus \\
\hline \multirow[t]{3}{*}{ Furst et al ${ }^{10}$} & 69 & $\mathrm{I} \mathrm{mg} /$ day & ACR20, ACR50 improvements & $29 \%$ (ACR20), I4.5\% (ACR50) \\
\hline & 64 & $3 \mathrm{mg} /$ day & & $34.4 \%$ (ACR20), 17.2\% (ACR50) \\
\hline & 64 & $5 \mathrm{mg} /$ day & & $50 \%$ (ACR20), $14.4 \%$ (ACR50) \\
\hline Kremer et $\mathrm{al}^{26}$ & 80 & $3 \mathrm{mg} /$ day & ACR20 improvements & $52.5 \%$ at 6 month \\
\hline Morita et $\mathrm{al}^{29}$ & 32 & $\mathrm{I}-3 \mathrm{mg} /$ day & EULAR criteria & $70 \%$ (moderate - good) at 6 month \\
\hline
\end{tabular}

joint counts, swelling joint counts, CRP, and general health score. ${ }^{29}$ Similar to our study, Furst DE reported that tender joints, ESR, and ACR 50 showed improvement in RA patients who were treated with the addition of tacrolimus at $1 \mathrm{mg}$ per day. ${ }^{10}$ Moreover, some studies that added low-dose tacrolimus to the treatment of patients who were non-responders to tumor necrosis factor inhibitor therapies and found improvements in RA. ${ }^{30,31}$ Clinical studies have demonstrated the efficacy of tacrolimus in RA patients with an insufficient response to MTX (summarized in Table 3). Thus, the addition of low-dose tacrolimus is thought to lead to improvement in rheumatoid arthritis. P-glycoprotein (P-gp), a member of the ATP-binding cassette transporter family, causes drug resistance via the exclusion of intracellular drugs. Tacrolimus, a P-gp inhibitor, can improve the effect of MTX by blocking P-gp. ${ }^{32}$

In conclusion, there were no significant side effects of the addition of tacrolimus to MTX therapy in terms of safety. Decreases were seen in the DAS28 (ESR) and mHAQ values, and it was also possible to reduce the dose of corticosteroids in two patients. Furthermore, the use of tacrolimus at only $1 \mathrm{mg}$ per day presents an extremely economical approach. By improving drug resistance, it is thought that the effect on the non-Japanese RA patients can be expected enough. We suggest that tacrolimus-MTX combination therapy be attempted prior to the administration of biological therapies.

\section{Disclosure}

No conflicts of interest were declared in relation to this paper.

\section{References}

1. Lee DM, Weinblatt ME. Rheumatoid arthritis. Lancet. 2001;358: 903-911.

2. Jenkins JK, Hardy KJ, McMurray RW. The pathogenesis of rheumatoid arthritis: a guide to therapy. Am J Med Sci. 2002;323:171-180.

3. Weinblatt ME. Efficacy of methotrexate in rheumatoid arthritis. Br J Rheumatol. 1995;34 Suppl 2:43-48.

4. Guidelines for the management of rheumatoid arthritis: 2002 Update. Arthritis Rheum. 2002;46:328-346.

5. Kino T, Hatanaka H, Hashimoto M, et al. FK-506, a novel immunosuppressant isolated from a Streptomyces. I. Fermentation, isolation, and physico-chemical and biological characteristics. JAntibiot (Tokyo). 1987; 40:1249-1255.
6. Sakuma S, Kato Y, Nishigaki F, et al. FK506 potently inhibits T cell activation induced TNF-alpha and IL-1beta production in vitro by human peripheral blood mononuclear cells. Br J Pharmacol. 2000; 130:1655-1663.

7. Yoshimura N, Matsui S, Hamashima T, Oka T. Effect of a new immunosuppressive agent, FK506, on human lymphocyte responses in vitro. II. Inhibition of the production of IL-2 and gamma-IFN, but not B cell-stimulating factor 2. Transplantation. 1989;47:356-359.

8. Yamagami T, Waguri-Nagaya Y, Ikuta K, Aoyama M, Asai K, Otsuka T. FK506 inhibition of gliostatin/thymidine phosphorylase production induced by tumor necrosis factor-alpha in rheumatoid fibroblast-like synoviocytes. Rheumatol Int. 2010 Mar 18. [Epub ahead of print]

9. Gremillion RB, Posever JO, Manek N, West JP, van Volen-Hoven RF. Tacrolimus (FK506) in the treatment of severe, refractory rheumatoid arthritis: initial experience in 12 patients. J Rheumatol. 1999;26: 2332-2336.

10. Furst DE, Saag K, Fleischmann MR, et al. Efficacy of tacrolimus in rheumatoid arthritis patients who have been treated unsuccessfully with methotrexate: a six-month, double-blind, randomized, dose-ranging study. Arthritis Rheum. 2002;46:2020-2028.

11. Suzuki K, Kameda H, Amano K, et al. Single center prospective study of tacrolimus efficacy and safety in treatment of rheumatoid arthritis. Rheumatol Int. 2009;29:431-436.

12. Suzuki K, Kameda H, Amano K, et al. Single center prospective study of tacrolimus efficacy and safety in the treatment of various manifestations in systemic lupus erythematosus. Rheumatol Int. 2010 Feb 19. [Epub ahead of print]

13. Niiyama S, Amoh Y, Suzuki K, Wada T, Katsuoka K. Efficacy of tacrolimus against Churg-Strauss syndrome in a patient with myasthenia gravis. Rheumatol Int. 2010;30:847-848.

14. Nozaki Y, Nagare Y, Kinoshita K, Urase F, Funauchi M. Successful treatment using tacrolimus plus corticosteroid in a patient with RA associated with MDS. Rheumatol Int. 2008;28:487-490.

15. Arnett FC, Edworthy SM, Bloch DA, et al. The American Rheumatism Association 1987 revised criteria for the classification of rheumatoid arthritis. Arthritis Rheum. 1988;31:315-324.

16. van der Heijde DM, van 't Hof MA, van Riel PL, et al. Judging disease activity in clinical practice in rheumatoid arthritis: first step in the development of a disease activity score. Ann Rheum Dis. 1990;49: 916-920.

17. van Gestel AM, Prevoo ML, van ‘t Hof MA, van Rijswijk MH, van de Putte LB, van Riel PL. Development and validation of the European League Against Rheumatism response criteria for rheumatoid arthritis. Comparison with the preliminary American College of Rheumatology and the World Health Organization/International League Against Rheumatism Criteria. Arthritis Rheum. 1996;39:34-40.

18. Ziebland S, Fitzpatrick R, Jenkinson C, Mowat A. Comparison of two approaches to measuring change in health status in rheumatoid arthritis: the Health Assessment Questionnaire (HAQ) and modified HAQ. Ann Rheum Dis. 1992;51:1202-1205.

19. Lubeck DP. Health-related quality of life measurements and studies in rheumatoid arthritis. Am J Manag Care. 2002;8:811-820.

20. Yamanaka H, MatsudaY, Tanaka M, et al. Serum matrix metalloproteinase 3 as a predictor of the degree of joint destruction during the six months after measurement, in patients with early rheumatoid arthritis. Arthritis Rheum. 2000;43:852-858. 
21. Ainola MM, Mandelin JA, Liljestrom MP, Li TF, Hukkanen MV, Konttinen YT. Pannus invasion and cartilage degradation in rheumatoid arthritis: involvement of MMP-3 and interleukin-1beta. Clin Exp Rheumatol. 2005;23:644-650.

22. Munster T, Furst DE. Pharmacotherapeutic strategies for diseasemodifying antirheumatic drug (DMARD) combinations to treat rheumatoid arthritis (RA). Clin Exp Rheumatol. 1999;17:S29-S36.

23. Capell HA, Madhok R, Porter DR, et al. Combination therapy with sulfasalazine and methotrexate is more effective than either drug alone in patients with rheumatoid arthritis with a suboptimal response to sulfasalazine: results from the double-blind placebo-controlled MASCOT study. Ann Rheum Dis. 2007;66:235-241.

24. Haagsma CJ, van Riel PL, de Jong AJ, van de Putte LB. Combination of sulphasalazine and methotrexate versus the single components in early rheumatoid arthritis: a randomized, controlled, double-blind, 52 week clinical trial. Br J Rheumatol. 1997;36:1082-1088.

25. Gerards AH, Landewe RB, Prins AP, et al. Cyclosporin A monotherapy versus cyclosporin $A$ and methotrexate combination therapy in patients with early rheumatoid arthritis: a double blind randomised placebo controlled trial. Ann Rheum Dis. 2003;62:291-296.

26. Kremer J, Genovese M, Cannon GW, et al. Combination leflunomide and methotrexate (MTX) therapy for patients with active rheumatoid arthritis failing MTX monotherapy: open-label extension of a randomized, double-blind, placebo controlled trial. J Rheumatol. 2004; 31:1521-1531.
27. Kasama T, Wakabayashi K, Odai T, et al. Effects of low-dose mizoribine pulse therapy in combination with methotrexate in rheumatoid arthritis patients with an insufficient response to methotrexate. Mod Rheumatol. 2009; $19: 395-400$.

28. Kremer JM, Habros JS, Kolba KS, et al. Tacrolimus in rheumatoid arthritis patients receiving concomitant methotrexate: a six-month, open-label study. Arthritis Rheum. 2003;48:2763-2768.

29. Morita Y, Sasae Y, Sakuta T, Satoh M, Sasaki T, Kashihara N. Efficacy of low-dose tacrolimus added to methotrexate in patients with rheumatoid arthritis in Japan: a retrospective study. Mod Rheumatol. 2008;18: 379-384.

30. Yokota K, Akiyama Y, Asanuma Y, Miyoshi F, Sato K, Mimura T. Efficacy of tacrolimus in infliximab-refractory progressive rheumatoid arthritis. Rheumatol Int. 2009;29:459-461.

31. Naniwa T, Watanabe M, Banno S, Maeda T. Adding low dose tacrolimus in rheumatoid arthritis patients with an inadequate response to tumor necrosis factor inhibitor therapies. Rheumatol Int. 2009;29: 1287-1291.

32. Tsujimura S, Saito K, Nawata M, Nakayamada S, Tanaka Y. Overcoming drug resistance induced by P-glycoprotein on lymphocytes in patients with refractory rheumatoid arthritis. Ann Rheum Dis. 2008;67: 380-388.
Open Access Rheumatology Research and Reviews

\section{Publish your work in this journal}

Open Access Rheumatology Research and Reviews is an international, peer-reviewed, open access journal, publishing all aspects of clinical and experimental rheumatology in the clinic and laboratory including the following topics: Pathology, pathophysiology of rheumatological diseases; Investigation, treatment and management of rheumatological

\section{Dovepress}

diseases; Clinical trials and novel pharmacological approaches for the treatment of rheumatological disorders. The manuscript management system is completely online and includes a very quick and fair peerreview system, which is all easy to use. Visit http://www.dovepress.com/ testimonials.php to read real quotes from published authors. 